\title{
Mathematical Modeling of Ultrasonic Phased Array for Obstacle Location for Visually Impaired
}

\author{
Ashwini Naik ${ }^{1}$, Dr M.S.Panse ${ }^{2}$ \\ ${ }^{1,2}$ (Electrical Engineering, V.J.T.I / Mumbai University, India)
}

\begin{abstract}
Ultrasonic beam steering characteristics of phased array transducer is mathematically modeled to detect and locate the obstacle for the visually impaired. The main purpose of Beam steering is to steer the beam at a given angle to investigate the object along the beam direction. The effects of various transducer parameters on beam steering properties are studied and optimal phased array is simulated. The device is constructed using $40 \mathrm{KHz}$ practically available ultrasonic sensors of $16 \mathrm{~mm}$ dimension. The main objective in designing is to minimize the main lobe width, to eliminate the grating lobes and to suppress the side lobes. In this paper the geometric parameters such as, number of elements, inter-element spacing, steering angle and array aperture are considered and transducer array is mathematically analyzed. The radiation pattern of the results is shown by the polar plots. These polar plots are simulated using LabVIEW (Laboratory virtual instrument engineering workbench) developed by National Instrument, is a graphical Programming environment suited for high-level or system level design. Advantage of this approach is the flexibility and very rapid development time offered by this graphical programming software.
\end{abstract}

Keywords: Grating lobes, LabVIEW, Radiation pattern, Ultrasonic Phased array

\section{INTRODUCTION}

During the past three decades, several researchers have introduced devices that use sensor technology to improve the blind user's mobility in terms of safety and speed. Examples of this device collectively are called Electronic Travel Aids (ETA's). An Electronic travel aid is a device that emits energy waves to detect within a certain range or distance, process reflected information and furnishes user with certain information about the immediate environment. Most of the devices rely on single beam, measuring only the distance to the obstacle. In order to locate the obstacle the user has to manually scan the area in front of him by pointing the device in relevant direction. The main motivation for this Design of ultrasonic phased array device stems from aiming to design a prototype, of Sensory aid for the visually impaired. For this application ultrasonic phased array sensors are used to transmit/receive sound waves in air. Ultrasonic sensors are widely regarded as simple and inexpensive device to sense the proximity of objects. Their ability to provide relatively accurate geometrical information concerning the existence, the localization, and the nature of insonified objects.

In most basic sense, a phased array system utilizes the wave physics principle of phasing [1], varying the time between a series of outgoing ultrasonic pulses in such a way that the individual wave fronts generated by each element in the array combine with each other to add or cancel energy in predictable ways that effectively steer and shape the sound beam. This is accomplished by pulsing the individual probe elements at slightly different times. The wave fronts in turn combine constructively and destructively in to single beam that travel in a particular direction. If there is an object in the path of this pulse, part or all of the pulse will be reflected back to the transmitter as an echo and can be detected through the receiver.

Array based systems have been used for various application, in radar, sonar, Medical ultrasound and NDT. The ultrasonic phased array principles have not been completely utilized to detect and locate the obstacle for visually impaired. To use ultrasonic phased array as obstacle detector, $40 \mathrm{kHz}$ probe is required to scan the area in front of the user at a distance of 1 to 2 meters. Since these devices are not practically available, construction becomes necessary. So in this work commercially available ultrasonic transducers of $40 \mathrm{kHz}$ frequency of $16 \mathrm{~mm}$ diameter are used to form transmitter array.

\section{DESIGN Of PhaSed ARRAY}

To design a phased array, it is very important to understand the wave propagation characteristics for various transducers parameters such as [2]

\subsection{Number of elements}

As the number of elements in an array increase, so can the physical coverage area of the probe and its sensitivity, focusing capability and steering capability. At the same time, use of large arrays must often be balanced against issues of system complexity and cost. 


\subsection{Pitch and aperture}

Pitch is the distance between individual elements, and aperture is the effective size of the pulsing element that is usually comprised of a group of individual elements that are pulsed simultaneously. To optimize steering range pitch must be small. For optimum sensitivity minimum unwanted beam spreading, and strong focusing, the aperture must be large.

\subsection{Grating lobes}

Grating lobes will occur whenever the size of individual element in an array is equal to or greater than the wavelength. The simplest way to minimize grating lobes is to use a transducer with smaller size $(<$ half wavelength). Specialized transducer design incorporates smaller element size and varying element spacing to reduce unwanted lobe.

\section{MODELLING OF ULTRASONIC PHASED ARRAY}

The operational principle of an ultrasonic phased array is identical phased array antenna[3] . The only difference is Antenna phased array transmit electromagnetic waves, whereas ultrasonic phased array transmit sound waves. Initial step in the design is to select number of elements in the transmitter array.

\subsection{Selecting number of elements in the transmitter array}

One of the key issues in designing an array transducer is to control the quality of the beam directivity and steer ability .i.e. the steered beams should be sharply defined and well-directed towards the desired steering direction. In the presence of obstacle, the reflected signal (echo) should be reproduced properly. The angular resolution required for proper reproduction of echo is considered to be 5 degrees.

Angular resolution $=$ Half power beam width of main beam (HPBW)

HPBW of a phased array is given by[3]

- $\mathrm{HPBW}($ degree $)=50.8 / \mathrm{a} / \lambda$

where ' $a$ ' aperture size $=\mathrm{Nxd}$,

$\mathrm{N}=$ number of elements, $\mathrm{d}=$ inter-element spacing

The size of transducer is $16 \mathrm{~mm}$ or $1.9 \lambda$. So minimum spacing between the transducers of the transmitter array can be $17 \mathrm{~mm}$ or $2 \lambda$. By substituting the values in equation (1) to get angular resolution less than $5^{\circ}$

$\mathrm{N}=5$ gives angular resolution of 5.08 degrees

$\mathrm{N}=6$ gives angular resolution of 4.23 degrees

\subsection{Beam Steering characteristic}

The transmitter is formed by 6 transducer elements. Each element is excited with a time delay for the steering angle $\theta$ is given by[1]

- $\Delta \mathrm{t}_{\mathrm{n}}=\mathrm{nd} / \mathrm{c}(\sin \theta)+\mathrm{t}$

where $d$ is the inter-element spacing, $c=$ velocity of sound in air $(340 \mathrm{~m} / \mathrm{s}), \mathrm{n}$ is the element number

The Ultrasonic signal generated can be represented mathematically [4] as

- $\quad \mathrm{S}(\mathrm{t})=\mathrm{At}^{\mathrm{m}} \mathrm{e}^{-\mathrm{t} / \mathrm{u}} \cos (2 \pi \mathrm{ft}+\Phi)$

The transmitted signal is as shown in Fig. 1. The signal is generated using $\mathrm{f}=40 \mathrm{kHz}, \mathrm{m}=2$ and $\mathrm{u}=3.6 \mathrm{E}-4$.

The time sequence of the excitation signal is calculated using equation (2). The inter-element distance is considered to be twice the wavelength. 


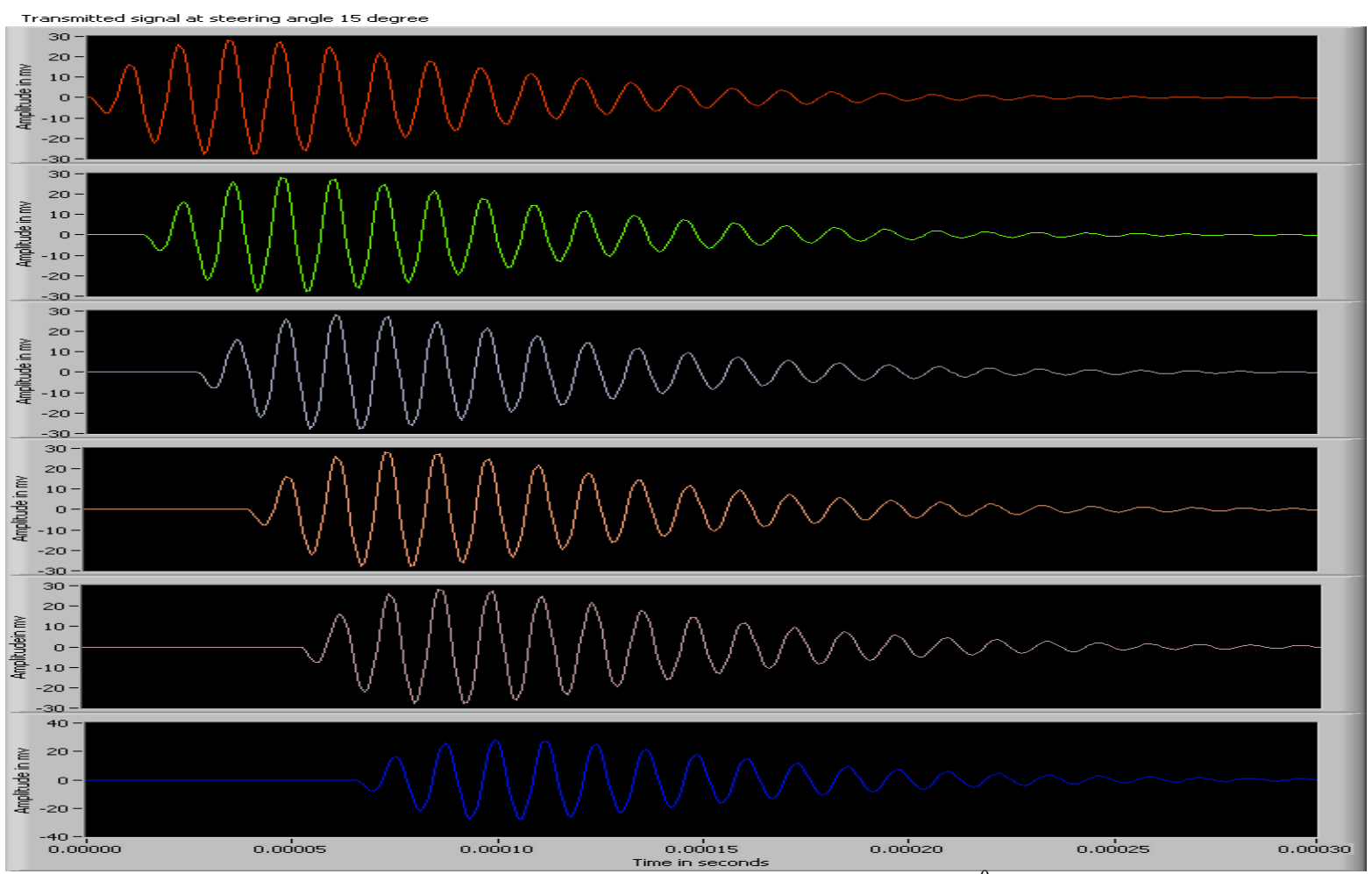

Figure 1. Front panel showing the ultrasonic wave for steering angle of $15^{0}$

\subsection{Grating lobes in the transmitter array}

The steered beam should be sharply defined and well directed towards the desired steering angle.

Radiation pattern of the transmitter is plotted with 6 elements of $2 \lambda$ inter-element. The magnitude of the main lobe, grating lobe from the equation (4)

$$
\text { - } \mathrm{D}(\theta)=\left|\frac{\frac{\pi a \sin \theta s}{\lambda}}{\sin \frac{\pi a \sin \theta s}{\lambda}} \frac{\sin \frac{\pi a \sin \theta}{\lambda}}{\frac{\pi a \sin \theta}{\lambda}}\right|\left|\frac{\sin \left(\frac{\pi \mathrm{d}(\sin \theta s-\sin \theta)}{\lambda}\right)}{N \sin \left(\frac{\pi d(\sin \theta s-\sin \theta)}{\lambda}\right)}\right|
$$

The complete Radiation pattern is given as the product of directivity of single element and directivity of the array [2]. From the above equation appearance of maximum lobe can be calculated using equation (5) [6][7]

- $\theta \mathrm{m}=\sin ^{-1}\left[\frac{1}{\mathrm{kd}}(-\beta+2 \mathrm{~m} \pi)\right]$

for $m= \pm 0,1.2 \ldots, \beta=$ phase delay between the elements and is calculated as

- $\beta=-k d \sin \theta s$ when $\mathrm{m}=0$ corresponds main beam, $\mathrm{m}= \pm 1$ presence of first grating lobe

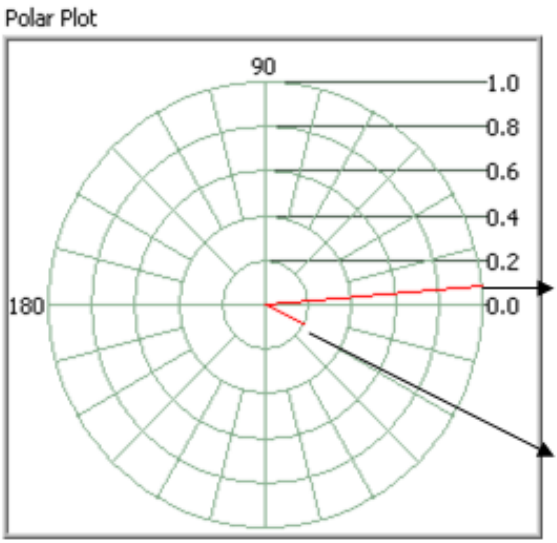

Figure 2a polar plot for steering angle $5^{\circ}$

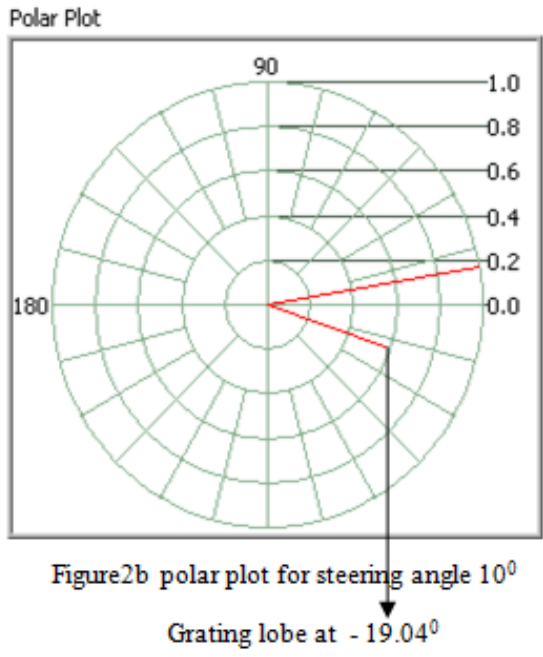

Www.iosrjournals.org 


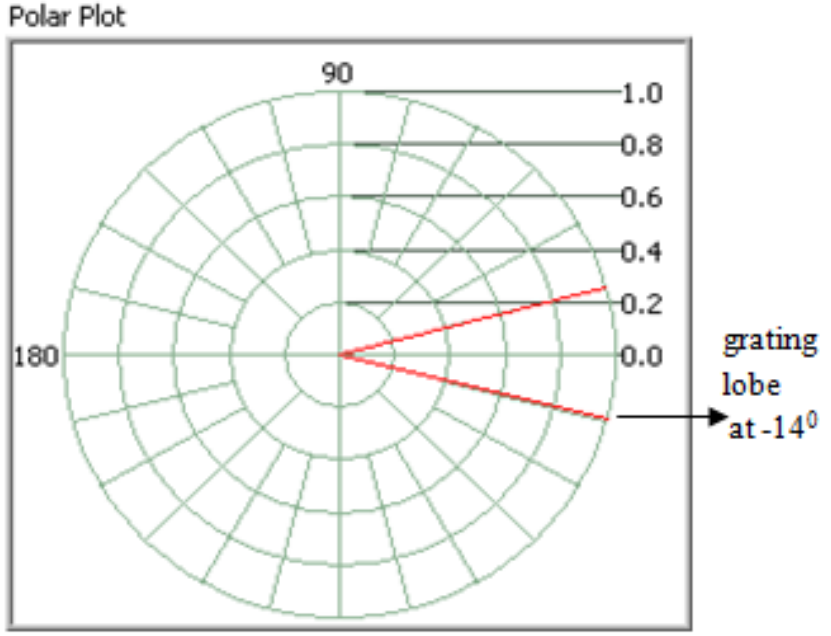

Figure 2c polar plot for steering angle $15^{\circ}$
Polar Plot

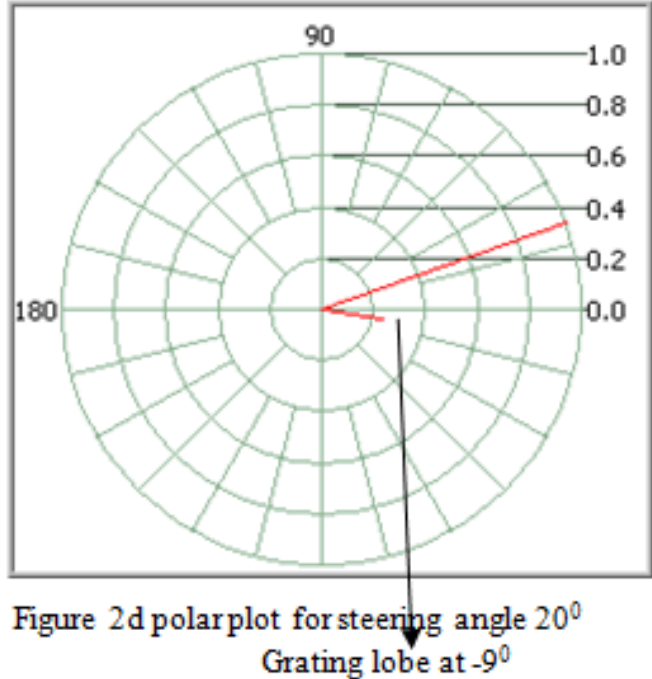

From the above polar plots it is seen that grating lobe follows the main lobe nearly by 29 degree. In case of 15 degree steering angle the grating lobe has the same magnitude as the main lobe, hence signal reproduction becomes complex. The effect of grating lobe can be reduced by adjusting the inter-element spacing of the receiver array so that the grating lobe align outside the scanning area. Here steering is performed from -20 degree to +20 degree, so that the grating lobes occuring at angles greater 20 degree and lesser than -20 degree are eliminated as they exist outside the scanning area. In between grating lobes can be reduced by varying the inter-element space of receiver array .

\subsection{Selection of number of elements in receiver array}

Grating lobes are undesirable and must be avoided. To avoid grating lobes $d / \lambda \leq 1 / 2$, or $d=\lambda / 2$. This is not possible as the size of transducer is $1.9 \lambda$. ' $d$ ' can be written as

- $\mathrm{d}=\mathrm{n} \lambda$ $\mathrm{n}=1,2,3$

For $n=1$ and $n=2$ spacing between receiver elements is not practically possible since transmitter width is greater than the wavelength, and if same spacing is placed between transmitter and receiver elements, grating lobes exist resulting in wrong location of obstacle. The minimum spacing can be for $n=3$,

- $\mathrm{d}=3 \lambda$.

Number of receiver elements can be calculated from half power bandwidth. From equation (1) $\mathrm{N}=4$ gives angular resolution of 4.23 degrees.

\subsection{Radiation pattern of receiver elements}

The receiver array is formed with 4 elements with inter-element spacing of $3 \lambda$. The effect of grating lobes of transmitter array can be minimized if the grating lobes of the transmitter array aligns with the nulls of the receiver array response. The nulls of the array[6] can be calculated from equation (6) given as,

- $\quad \theta \mathrm{n}=\sin ^{-1}\left[\frac{1}{\mathrm{kd}}\left(-\beta+\frac{2 \mathrm{n} \pi}{\mathrm{N}}\right)\right]$

where $\mathrm{n}= \pm 1,2,3 \ldots ., \quad \mathrm{n} \neq \mathrm{N}, 2 \mathrm{~N}, 3 \mathrm{~N} \ldots \ldots$

The nulls for the receiver array for steering angle 10, 15 and 20 degrees are as shown in the fig 3 . 


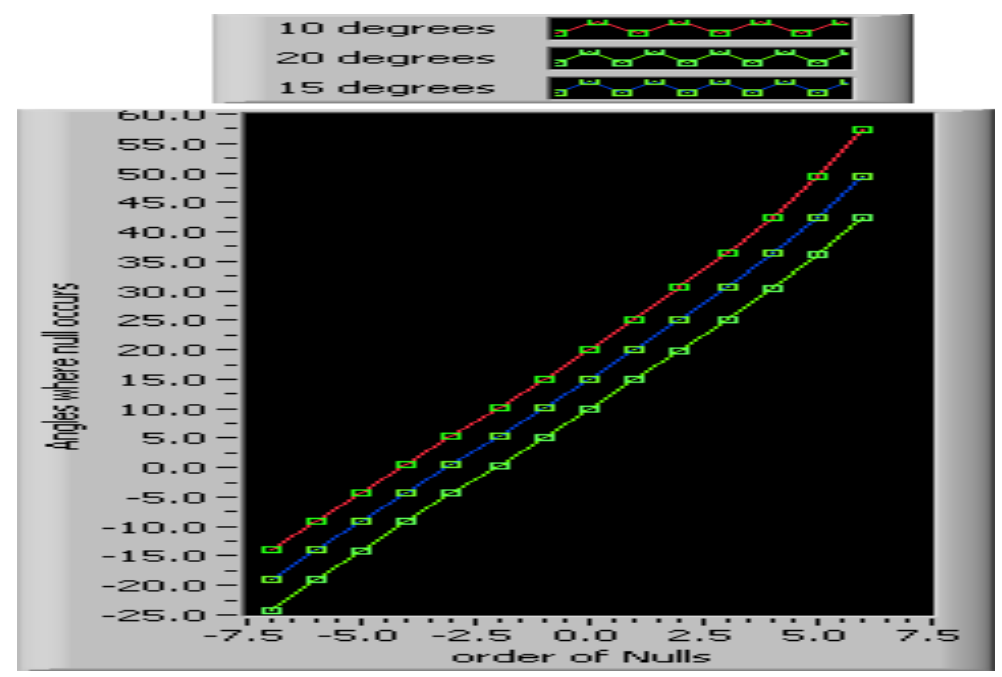

Figure 3 Graph showing nulls in the receiver array

IV.

\section{RESULTS}

The beam directivity pattern of the Transmitter array for different steering angles is as shown in fig. 2a, $2 \mathrm{~b}, 2 \mathrm{c}$ and $2 \mathrm{~d}$. There exists grating lobe for every particular steering angle calculated from equation (5) when $\mathrm{m}=-1$. For $\mathrm{m}=1$ in all the cases the grating lobe occurs far away from the field of view range. The graph in Fig.(3) shows that for steering angle of $15^{\circ}$, null exists at -14 degree for the order -6 . Similarly null occurs at 19 degree for steering angle of $10^{\circ}$.

\section{CONCLUSION}

Ultrasonic phased array device to locate obstacle for visually impaired is designed with the phased array antenna concepts. The transmitter array of 6 elements with $2 \lambda$ inter-element spacing and receiver array of 4 elements with $3 \lambda$ spacing. The transducers used for construction are $40 \mathrm{khs}$ frequency and $16 \mathrm{~mm}(1.9 \lambda)$ in dimension. Since the width of the transducer is greater than the wavelength, grating lobes were sure to exist. The array model designed here reduces the grating lobe and gives optimum steering characteristics. The designed device is simple,compact and ability to scan which fulfils the conditions of developing a prototype travelling device for visually impaired people.

\section{References}

[1] O.T. von Ramm and S.W. Smith. : Beam steering with linear arrays: IEEE Transactions on Biomedical Engineering, vol. BME-30(8), pp. 438-452.1983.

[2] Shi-Chang Wooh and Yijun Shi. : A Simulation Study of the Beam Steering Characteristics for Linear Phased Arrays: Journal of Nondestructive Evaluation, 18(2), pp 39-57,1999.

[3] Alfred Hanssen, Wavefields, in Simon Haykin, 1(Wiley 2010), Handbook on Array Processing and Sensor Networks, 5-30.

[4] Theodore C. Cheston, Phased Array Radar Antennas in Merrill I. Skolnik, Radar Handbook, 7(McGraw-Hill,1990 ), 7.1-7.16.

[5] J.Huang, P.W.Que, and J.H.Jin, A Parametric study of Beam steering for ultrasonic linear phased array transducer, Russian Journal of Nondestructive Testing, 40(4), pp254-259,2004.

[6] C.A. Balanis, Antenna Theory: Analysis and Design, (John Wiley and Sons, NewYork, 1997).

[7] Sevan Harput, Ayhan Bozkurt, Ultrasonic Phased Array Device for Acoustic Imaging in Air, IEEE Sensors Journal, l 8(11), pp 17551762,2008 